\title{
NONUNIFORM DICHOTOMY OF EVOLUTIONARY PROCESSES IN BANACH SPACES
}

\author{
Petre Preda and Minail Megan
}

\begin{abstract}
In this paper we study nonuniform dichotomy concepts of linear evolutionary processes which are defined in a general Banach space and whose norms can increase no faster than an exponential. Connections between the dichotomy concepts and $(B, D)$ admissibility properties are established. These connections have been partially accomplished in an earlier paper by the authors for the case when the process was a semigroup of class $C_{0}$ and $(B, D)=\left(L^{p}, L^{q}\right)$
\end{abstract}

\section{Introduction}

The dichotomy concepts for linear differential equations and their connections with admissibility properties have been extensively studied among others by Coppel [1] and Massera and Schäffer [3]. The study of the asymptotical behaviour of linear time-varying systems underlines nonuniform stability and nonuniform dichotomy properties (see for example [3], [5], [1] and [8]). Nonuniform dichotomic behaviours for the general case when the evolution of the system is described by a linear evolutionary process $P(\cdot, \cdot)$ on a general Banach space are considered in this paper. Using a fundamental inequality established in [3] we define the concept of $(B, D)$ dichotomic evolutionary process and give a sufficient condition for nonuniform exponential dichotomy of a large class of such processes. We also consider the connections between the dichotomy concepts and the $(B, D)$

Received 2 August 1982. 
admissibility property for the case of a linear system described by

$$
x\left(t, t_{0}, x_{0}, u\right)=P\left(t, t_{0}\right) x_{0}+\int_{t_{0}}^{t} P(t, s) u(s) d s .
$$

The case when $P\left(t, t_{0}\right)=P\left(t-t_{0}, 0\right)$, that is, $P(\cdot, \cdot)$ is a $C_{0}-$ semigroup, has been considered in [5]. The obtained results may be regarded as generalizations of well-known results of Coppel, Massera and Schäffer, Palmer and Reghis. They are applicable for a large class of systems described in [2].

Thus this paper is in a sense a sequel to [5].

\section{Notation, definitions and terminology}

Let $(X,\|\cdot\|)$ be a real or complex Banach space. The space of continuous linear mappings from $X$ into itself is denoted by $L(X)$. The symbol $\Delta$ will denote the set defined by

$$
\Delta=\{(t, s): 0 \leq s \leq t<\infty\} \text {. }
$$

DEFINITION 2.1. An application $P(\cdot, \cdot): \Delta \rightarrow L(X)$ will be called an evolutionary process if and only if

(i) $P(t, s) P\left(s, t_{0}\right)=P\left(t, t_{0}\right)$ for $0 \leq t_{0} \leq s \leq t$,

(ii) $P(t, t) x=x$ for every $x \in X$,

(iii) $P(t, s)$ is strongly continuous in $s$ on $[0, t]$ and in $t$ on $[s, \infty)$,

(iv) there exists a nondecreasing function

$$
p: \mathbf{R}_{+}=[0, \infty) \rightarrow \mathbf{R}_{+}=(0, \infty)
$$

such that

$\|P(t, s) x\| \leq p(t-s)\|x\|$ for all $(t, s) \in \Delta$ and $x \in X$.

REMARK 2.1. If $P(\cdot, \cdot)$ is an evolutionary process then its norm can increase no faster than an exponential, that is, there exist $M, \omega>0$ such that$$
\|P(t, s)\| \leq M e^{\omega(t-s)} \text { for all }(t, s) \in \Delta \text {. }
$$$$
\text { Indeed, if } M=p(1), \omega=\ln M \text { and } n \text { is the positive integer such }
$$ 
that $n \leq t-s<n+1$ then

$$
\begin{aligned}
\|P(t, s)\| & \leq\|P(t, s+n)\| \cdot\|P(s+n, s+n-1)\| \cdot \ldots \cdot\|P(s+1, s)\| \\
& \leq M e^{n \omega} \\
& \leq M e^{\omega(t-s)} .
\end{aligned}
$$

REMARK 2.2. If the evolutionary process $P(\cdot, \cdot)$ satisfies the condition

(v) $P(t, s)=P(t-s, 0)$ for all $(t, s) \in \Delta$, then $P(\cdot, \cdot)$ is a semigroup of class $C_{0}$.

The space of $X$-valued functions $f$ almost defined on $\mathbb{R}_{+}$such that $f$ is strongly measurable and locally integrable is denoted by $L_{l o c}^{l}(X)$. In particular $L_{10 c}^{l}(\mathbb{R})=L_{\text {loc }}^{1}$. If $I=[a, b]$ is a real compact interval, then the characteristic function of $I$ will be denoted by $\varphi_{a}^{b}$. In the particular cases $a=0$ and respectively $b=\infty$ we use the notation

$$
\varphi_{0}^{b}=\varphi^{b} \text { and respectively } \varphi_{a}^{\infty}=\varphi_{a}
$$

DEFINITION 2.2. A Banach space $\left(S,|\cdot|_{S}\right)$ is said to be a Schäffer space (and we write $S \in S$ ) if it has the following properties:

(i) $S \subset L_{\text {loc }}^{1}$ and there exists $M>0$ such that

$$
\int_{0}^{t}|f(s)| d s \leq M \cdot|f|_{S} \text { for every } S \in S \text { and } t \geq 0 \text {; }
$$

(ii) if $g \in S$ and $f$ is a real measurable function with $|f| \leq g$ almost everywhere on $\mathbb{R}_{+}$then $f \in S$ and $|f|_{S} \leq|g|_{S}$

(iii) for every $f \in S$ and $t \geq 0$ the function

$$
g_{t}(s)= \begin{cases}0 & , \text { if }(t, s) \in \Delta, \\ f(s-t), & \text { if }(s, t) \in \Delta,\end{cases}
$$

is also in $s$ with $\left|g_{t}\right|_{S}=|f|_{S}$; 
(iv) for every $t \geq 0$ the function

$$
\varphi^{t}(s)=\left\{\begin{array}{l}
1, \text { if }(t, s) \in \Delta, \\
0, \text { if }(t, s) \notin \Delta,
\end{array}\right.
$$

is in $S$.

REMARK 2.3. It is obvious that the following Banach spaces are Schäffer spaces :

(i) ${ }_{L}^{p}$ (where $1 \leq p<\infty$ ), the space of real $p$-integrable functions $f$ on $\mathrm{R}_{+}$with the norm

$$
\left.\|f\|_{p}=\iint_{\mathbf{R}_{+}}|f(s)|^{p} d s\right)^{1 / p}
$$

(ii) $L^{\infty}$, the space of essentially bounded real measurable functions $f$ with the norm

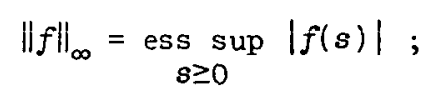

(iii) $M^{p}$ (with $1 \leq p<\infty$ ), the space of real measurable functions $f$ such that

$$
|f|_{p}=\sup _{t \geq 0}\left(\int_{t}^{t+1}|f(s)|^{p} d s\right)^{1 / p}<\infty ;
$$

(iv) $M^{\infty}=L^{\infty}$ with the norm $|\cdot|_{\infty}=\|\cdot\|_{\infty}$;

(v) $C$, the space of real bounded and continuous functions $f$ defined on $\mathbb{R}_{+}$with the norm

$$
\left\|f\left|\|=\sup _{t \geq 0}\right| f(t) \mid\right. \text {. }
$$

If we denote by $S(X)$ (where $S \in S$ ) the space of $X$-valued functions $f$ almost defined on $R_{+}$, such that $f$ is strongly measurable and $\|f\| \in S$ then $S(X)$ is a Banach space with the norm $\|f\|_{S}$, which we write without ambiguity as $\|f\|_{S}$.

For every $S \in S$ we associate the functions $\alpha_{S}, \beta_{S}: \mathbf{R}_{+} \rightarrow \mathbf{R}_{+}$ defined by 


$$
\alpha_{S}(t)=\inf \left\{M>0 ; \int_{0}^{t}|f(s)| d s \leq M|f|_{S}, \forall f \in S\right\}
$$

and respectively

$$
\beta_{S}(t)=\left|\varphi^{t}\right|_{S}
$$

REMARK 2.4. In [3, pp. 61-67] it is shown that the function $\alpha_{S}$ and $\beta_{S}$ have the following properties:

(i) $t \leq \alpha_{S}(t) \beta_{S}(t) \leq 2 t$ for all $t \geq 0$ and $S \in S$;

(ii) if $t_{1} \leq t_{2}$ then $\alpha_{S}\left(t_{1}\right) \leq \alpha_{S}\left(t_{2}\right)$ and $\beta_{S}\left(t_{1}\right) \leq \beta_{S}\left(t_{2}\right)$;

(iii) $\alpha_{S}(t)=\inf \left\{M>0: \int_{a}^{b}|f(s)| d s \leq M|f|_{S}, \forall f \in S\right\}$ for all $a, b>0$ with $b-a=t$.

If $S \in S$ and $t_{0}>0$ then we denote by $S_{t_{0}}(X)$ the space of functions $f:\left[t_{0}, \infty\right) \rightarrow X$ with the property that $\tilde{f}: \mathbb{R}_{+} \rightarrow X$ defined by

$$
\tilde{f}(t)=\left\{\begin{array}{lll}
f(t) & , \text { if } & \left(t, t_{0}\right) \in \Delta, \\
0 & , \text { if } & \left(t, t_{0}\right) \notin \Delta,
\end{array}\right.
$$

is in $S(X)$.

Throughout in this paper we suppose that for all $D \in S$ and $t_{0} \geq 0$ the set

$$
X_{1}^{D}\left(t_{0}\right)=\left\{x_{0} \in X: P\left(\cdot, t_{0}\right) x_{0} \in D_{t_{0}}(X)\right\}
$$

is a closed complemented subspace.

If $x_{2}^{D}\left(t_{0}\right)$ is a subspace such that

$$
x=x_{1}^{D}\left(t_{0}\right) \oplus x_{2}^{D}\left(t_{0}\right)
$$

then we denote by $P_{1}\left(t_{0}\right)$ the projection on to $x_{2}^{D}\left(t_{0}\right)$ (that is 
$\operatorname{Ker} P_{1}\left(t_{0}\right)=x_{1}^{D}\left(t_{0}\right)$ ) and we let $P_{2}\left(t_{0}\right)=I-P_{1}\left(t_{0}\right)$ (where $I$ is the identity operator on $X)$, which is the projection on to $x_{1}^{D}\left(t_{0}\right)$.

We shall let

$$
P_{1}\left(t, t_{0}\right)=P\left(t, t_{0}\right) P_{1}\left(t_{0}\right)
$$

and

$$
P_{2}\left(t, t_{0}\right)=P\left(t, t_{0}\right) P_{2}\left(t_{0}\right)
$$

Now let us note two assumptions which will be used at various times.

ASSUMPTION 1. Let $B, D \in S$. We say that the pair $(B, D)$ satisfies Assumption 1 if

$$
\lim _{t \rightarrow \infty} \alpha_{B}(t) B_{D}(t)=\infty
$$

ASSUMPTION 2. The evolutionary process $P(\cdot, \cdot)$ satisfies Assumption 2 if there are two applications $m, \delta: \mathbb{R}_{+} \rightarrow \mathbb{R}_{+}^{*}$ such that

$$
\left\|P_{2}\left(t+\delta\left(t_{0}\right), t_{0}\right) x\right\| \geq m\left(t_{0}\right)\left\|P_{2}\left(t, t_{0}\right) x\right\|
$$

for all $\left(t, t_{0}\right) \in \Delta$ and $x \in X$.

DEFINITION 2.3. Let $P(\cdot, \cdot)$ be an evolutionary process. Then $P(\cdot, \cdot)$ is said to be

(i) a (nonuniformly) exponentially dichotomic process if and only if there are $N, v: \mathbb{R}_{+} \rightarrow \mathbb{R}_{+}$such that

$$
\left\|P_{1}\left(t, t_{0}\right) x\right\| \leq N\left(t_{0}\right) e^{-v\left(t_{0}\right)(t-s)}\left\|P_{1}\left(s, t_{0}\right) x\right\|
$$

and

$$
\left\|P_{2}\left(t, t_{0}\right) x\right\| \geq N\left(t_{0}\right) e^{v\left(t_{0}\right)(t-s)}\left\|P_{2}\left(s, t_{0}\right) x\right\|
$$

for all $t \geq s \geq t_{0} \geq 0$ and $x \in X$;

(ii) a (nonumiformly) dichotomic process if and only if there exists $N: \mathbb{R}_{+} \rightarrow \mathbb{R}_{+}$such that 


$$
\left\|P_{1}\left(t, t_{0}\right) x\right\| \leq N\left(t_{0}\right)\left\|P_{1}\left(s, t_{0}\right) x\right\|
$$

and

$$
\left\|P_{2}\left(t, t_{0}\right) x\right\| \geq N\left(t_{0}\right)\left\|P_{2}\left(s, t_{0}\right) x\right\|
$$

for all $t \geq s \geq t_{0} \geq 0$ and $x \in X$.

REMARK 2.5. It is obvious that

(i) if $P(\cdot, \cdot)$ is an exponentially dichotomic process it is dichotomic,

(ii) if $P(\cdot, \cdot)$ is a dichotomic process then Assumption 2 holds.

REMARK 2.6. Let us denote

$$
X_{1}\left(t_{0}\right)=\left\{x_{0} \in X: P\left(\cdot, t_{0}\right) x_{0} \in L_{t_{0}}^{\infty}(X)\right\} .
$$

(i) If $P(\cdot, \cdot)$ is a dichotomic process then $X_{1}^{D}\left(t_{0}\right) \subset X_{1}\left(t_{0}\right)$. Indeed, if $x_{0} \in X_{1}^{D}\left(t_{0}\right)$ then

$$
\left\|P\left(t, t_{0}\right) x_{0}\right\| \leq N\left(t_{0}\right)\left\|x_{0}\right\|
$$

for every $t \geq t_{0}$ and hence $x_{0} \in X_{1}\left(t_{0}\right)$.

(ii) If $P(\cdot, \cdot)$ is an exponentially dichotomic process then

$$
X_{1}^{D}\left(t_{0}\right)=X_{1}\left(t_{0}\right) \text { for all } D \in S \text { and } t_{0} \geq 0 \text {. }
$$

For this, it is sufficient to observe that if $x_{0} \in X_{1}\left(t_{0}\right)$ and $P_{2}\left(t_{0}\right) x_{0} \neq 0$ then

$$
\begin{aligned}
\left\|P\left(t, t_{0}\right) x_{0}\right\| \\
\quad \geq\left\|P_{2}\left(t, t_{0}\right) x_{0}\right\|-\left\|P_{1}\left(t, t_{0}\right) x_{0}\right\| \\
\quad \geq N\left(t_{0}\right) e^{v\left(t_{0}\right)\left(t-t_{0}\right)} \cdot\left\|P_{2}\left(t_{0}\right) x_{0}\right\|-N\left(t_{0}\right) e^{-v\left(t_{0}\right)\left(t-t_{0}\right)} \cdot\left\|P_{1}\left(t_{0}\right) x_{0}\right\|,
\end{aligned}
$$

implies that

$$
\lim _{t \rightarrow \infty}\left\|P\left(t, t_{0}\right) x_{0}\right\|=\infty
$$


wich contradicts the fact that $x_{0} \in X_{1}\left(t_{0}\right)$.

DEFINITION 2.4. Let $B, D$ be two Schäffer spaces. The evolutionary process $P(\cdot, \cdot)$ is $(B, D)$ dichotomic if and only if there exists a function $N: R_{+} \rightarrow R_{+}$such that

$$
\left\|\varphi_{t+\delta}(\cdot) P_{1}\left(\cdot, t_{0}\right) x\right\|_{D}+\left\|\varphi \varphi^{t}(\cdot) P_{2}\left(\cdot, t_{0}\right) x\right\|_{D} \leq \frac{N\left(t_{0}\right)}{\delta \alpha_{B}(\delta)} \cdot \int_{t}^{t+\delta}\left\|P\left(s, t_{0}\right) x\right\| d s
$$

for all $\left(t, t_{0}\right) \in \Delta, \delta>0$ and $x \in X_{k}\left(t_{0}\right), k=1,2$.

Consider the linear system described by the following integral model

$$
x\left(t, t_{0}, x_{0}, u\right)=P\left(t, t_{0}\right) x_{0}+\int_{t_{0}}^{t} P(t, s) u(s) d s,
$$

where $\left(t, t_{0}\right) \in \Delta, x_{0} \in X, u \in B(X)$.

DEFINITION 2.5. Let $B, D$ be two Schäffer spaces. We say that the pair $(B, D)$ is admissible for the system $(P)$ if and only if for all $t_{0} \geq 0$ and $u_{0} \in B_{t_{0}}(X)$ there exists $x_{0} \in X$ such that $x\left(\cdot, t_{0}, x_{0}, u_{0}\right) \in D_{t_{0}}(X)$.

REMARK 2.7. If the pair $\left(B, L^{\infty}\right)$ is admissible for the system (P) and

$$
\lim _{t \rightarrow \infty} \alpha_{B}(t)=\infty
$$

then (see Corollary 4.2) Assumption 2 holds.

\section{Preliminary results}

We prove the following lemmas which will be used in the sequel.

LEMMA 3.1. If $f: \Delta \rightarrow R_{+}$is a fionction with the property that there is $\delta: \mathbf{R}_{+} \rightarrow \mathbf{R}_{+}^{*}$ such that $2 f\left(t+\delta\left(t_{0}\right), t_{0}\right) \leq f\left(t, t_{0}\right) \leq 2 f\left(s, t_{0}\right)$ for all $s+\delta\left(t_{0}\right) \geq t \geq s \geq t_{0} \geq 0$ then there exists $v: \mathbb{R}_{+}^{*} \rightarrow \mathbb{R}_{+}^{*}$ such that

$$
f\left(t, t_{0}\right) \leq 4 e^{-v\left(t_{0}\right)(t-s)} f\left(s, t_{0}\right)
$$


for all $t \geq s \geq t_{0} \geq 0$.

Proof. If $t \geq s \geq t_{0} \geq 0$ and $n$ is the positive integer such that $n \delta \leq t-s<(n+1) \delta$ then

$$
\begin{aligned}
f\left(t, t_{0}\right) & \leq 2 f\left(s+n \delta\left(t_{0}\right), t_{0}\right) \\
& \leq 2\left(\frac{2}{2}\right)^{n} f\left(s, t_{0}\right) \\
& =4 e^{-(n+1) v\left(t_{0}\right) \delta(t)} \cdot f\left(s, t_{0}\right) \\
& \leq 4 e^{-v\left(t_{0}\right)(t-s)} \cdot f\left(s, t_{0}\right),
\end{aligned}
$$

where $v\left(t_{0}\right)=\ln 2 / \delta\left(t_{0}\right)$.

LEMMA 3.2. Let $f: \Delta \rightarrow \mathbb{R}_{+}$be a function with the property that there is $\delta: \mathbb{R}_{+} \rightarrow \mathbb{R}_{+}^{*}$ such that $f\left(s, t_{0}\right) \leq 2 f\left(t, t_{0}\right) \leq f\left(t+\delta\left(t_{0}\right), t_{0}\right)$ for all $s+\delta\left(t_{0}\right) \geq t \geq s \geq t_{0} \geq 0$ then there is $\nu: \mathbb{R}_{+} \rightarrow \mathbb{R}_{+}^{*}$ such that

$$
4 f\left(t, t_{0}\right) \geq e^{v\left(t_{0}\right)(t-s)} \cdot f\left(s, t_{0}\right)
$$

for all $t \geq s \geq t_{0} \geq 0$.

Proof. This is similar to the proof of the preceding lemma.

LEMMA 3.3. Let $(B, D)$ be a pair of Schäffer spaces which satisfy Assumption 1. If the evolutionary process $P(\cdot, \cdot)$ is $(B, D)$ dichotomic then there exists an application $n: \mathbb{R}_{+} \rightarrow \mathbb{R}_{+}^{*}$ such that

(i) $2 \int_{t}^{t+\delta}\left\|P_{1}\left(\tau, t_{0}\right) x\right\| d \tau \leq \int_{s}^{s+\delta}\left\|P_{1}\left(\tau, t_{0}\right) x\right\| d \tau$ for alz $s \geq t_{0} \geq 0, \delta \geq n\left(t_{0}\right), \quad t \geq s+n\left(t_{0}\right)$ and $x \in X$, and

(ii) $2 \int_{s}^{s+\delta}\left\|P_{2}\left(\tau, t_{0}\right) x\right\| d \tau \leq \int_{t}^{t+\delta}\left\|P_{2}\left(\tau, t_{0}\right) x\right\| d \tau$ for all

$$
s \geq t_{0} \geq 0, \delta>n\left(t_{0}\right), \quad t \geq s+2 n\left(t_{0}\right) \text { and } x \in X .
$$

Proof. If $P(\cdot, \cdot)$ is a $(B, D)$ dichotomic process then there exists an application $N: \mathbf{R}_{+} \rightarrow R_{+}$such that the inequality from Definition 2.4 holds. 
For every $t_{0} \geq 0$ let $\delta_{0}=n\left(t_{0}\right)>0$ be sufficiently large to satisfy

$$
\frac{8 N\left(t_{0}\right)}{\alpha_{B}\left(\delta_{0}\right) \beta_{D}\left(\delta_{0}\right)}<1
$$

Let now $s \geq t_{0} \geq 0, \delta \geq \delta_{0}$ and let $n$ be the positive integer such that $n \delta_{0} \leq \delta<(n+1) \delta_{0}$. If we denote by $\delta_{1}=\delta / n$ then for $t \geq s+\delta_{0}$ and $\tau=s+k \delta_{1}, k=0,1, \ldots, n-1$ we obtain

$$
\begin{aligned}
\int_{\tau+t-s}^{\tau+t-s+\delta_{1}\left\|P_{1}\left(u, t_{0}\right) x\right\| d u} & \leq \alpha_{D}\left(\delta_{1}\right)\left\|\varphi_{\tau+\delta_{0}}(\cdot) P_{1}\left(\cdot, t_{0}\right) x\right\|_{D} \\
& \leq \frac{N\left(t_{0}\right) \alpha_{D}\left(\delta_{1}\right)}{\delta_{0} \alpha_{B}\left(\delta_{0}\right)} \cdot \int_{\tau}^{\tau+\delta_{0}}\left\|P_{1}\left(u, t_{0}\right) x\right\| d u \\
& \leq \frac{2 N\left(t_{0}\right) \delta_{1}}{\delta_{0} \alpha_{B}\left(\delta_{0}\right) \beta_{D}\left(\delta_{1}\right)} \cdot \int_{\tau}^{\tau+\delta_{0}}\left\|P_{1}\left(u, t_{0}\right) x\right\| d u \\
& \leq \frac{4 N\left(t_{0}\right)}{\alpha_{B}\left(\delta_{0}\right) \beta_{D}\left(\delta_{0}\right)} \cdot \int_{\tau}^{\tau+\delta_{0}} \| P_{1}\left(u, t_{0}\right) x i d u \\
& \leq \frac{3}{2} \int_{\tau}^{\tau+\delta_{0}}\left\|P_{1}\left(u, t_{0}\right) x\right\| d u .
\end{aligned}
$$

Taking $\tau=s+k \delta_{1}, k=0,1,2, \ldots, n-1$ and adding we obtain

$$
\int_{t}^{t+\delta}\left\|P_{1}\left(u, t_{0}\right) x\right\| d u \leq \frac{1}{2} \int_{s}^{s+\delta}\left\|P_{1}\left(u, t_{0}\right) x\right\| d u
$$

which proves the inequality (i).

For the proof of (ii) let $s \geq t_{0}, t \geq s+2 \delta_{0}$ and $\tau=s+k \delta_{1}$ with $k=0,1, \ldots, n-1$. Then as before we obtain

$$
\begin{aligned}
\int_{\tau}^{\tau+\delta} 1\left\|P_{2}\left(u, t_{0}\right) x\right\| d u & \leq \alpha_{D}(\delta)\left\|\varphi^{\tau+s}(\cdot) P_{2}\left(\cdot, t_{0}\right) x\right\|_{D} \\
& \leq \frac{3}{2} \int_{\tau+t-s}^{\tau+t-s+\delta} 1\left\|P_{2}\left(u, t_{0}\right) x\right\| d u
\end{aligned}
$$


and adding, we obtain the inequality ( $i i)$.

LEMMA 3.4. Let $(B, D)$ be a pair of Schäffer spaces which satisfy Assumption 1. If the evolutionary process $P(\cdot, \cdot)$ is $(B, D)$ dichotomic then there are $v: \mathbb{R}_{+} \rightarrow \mathbb{R}_{+}^{*}, M: \mathbb{R}_{+} \times \mathbb{R}_{+}^{*} \rightarrow \mathbb{R}_{+}^{*}$ such that

$$
\begin{aligned}
& \text { (i) } \int_{t}^{t+\delta}\left\|P_{1}\left(u, t_{0}\right) x\right\| d u \leq M\left(t_{0}, \delta\right) e^{-v\left(t_{0}\right)(t-s)}\left\|P_{1}\left(s, t_{0}\right) x\right\| \text { and } \\
& \text { (ii) } \int_{s}^{s+\delta}\left\|P_{2}\left(u, t_{0}\right) x\right\| d u \leq M\left(t_{0}, \delta\right) e^{-v\left(t_{0}\right)(t-s)}\left\|P_{2}\left(t, t_{0}\right) x\right\|
\end{aligned}
$$

for all $t \geq s \geq t_{0} \geq 0, \delta>0$ and $x \in X$.

Proof. Let $n(\cdot)$ be the function defined in the preceding theorem. For $\delta>0$ and $x \in X$ consider the function

$$
f: \Delta \rightarrow \mathbb{R}_{+}, f\left(t, t_{0}\right)=\int_{t}^{t+n \delta}\left\|P_{1}\left(u, t_{0}\right) x\right\| d u,
$$

where $n=n\left(t_{0}, \delta\right)$ is sufficiently large such that $n \delta>4 n\left(t_{0}\right)$.

From the preceding lemma we have

$$
2 f\left(t+n\left(t_{0}\right), t_{0}\right) \leq f\left(t, t_{0}\right)
$$

and for $s+n\left(t_{0}\right) \geq t \geq s \geq t_{0} \geq 0$ the following inequalities hold:

$$
\begin{aligned}
f\left(t, t_{0}\right) & \leq \int_{s}^{s+n \delta+n\left(t_{0}\right)}\left\|P_{1}\left(u, t_{0}\right) x\right\| d u \\
& \leq \int_{s}^{s+n\left(t_{0}\right)}\left\|P_{1}\left(u, t_{0}\right) x\right\| d u+\int_{s}^{s+n \delta}\left\|P_{1}\left(u, t_{0}\right) x\right\| d u \\
& \leq \frac{3}{2} \cdot f\left(s, t_{0}\right) \\
& \leq 2 f\left(s, t_{0}\right) .
\end{aligned}
$$

By Lemma 3.1 it follows that there is $\nu: \mathbb{R}_{+} \rightarrow \mathbb{R}_{+}^{*}$ such that 


$$
\begin{aligned}
\int_{t}^{t+\delta}\left\|P_{1}\left(u, t_{0}\right) x\right\| d u & \leq f\left(t, t_{0}\right) \\
& \leq 4 e^{-v\left(t_{0}\right)(t-s)} f\left(s, t_{0}\right) \\
& \leq 4 e^{-v\left(t_{0}\right)(t-s)} \cdot \int_{s}^{s+n \delta}\|P(u, s)\| d u \cdot\left\|P_{1}\left(s, t_{0}\right) x\right\| \\
& \leq M\left(t_{0}, \delta\right)^{-v\left(t_{0}\right)(t-s)} \cdot\left\|P_{1}\left(s, t_{0}\right) x\right\|,
\end{aligned}
$$

where $M: \mathbb{R}_{+} \times \mathbb{R}_{+}^{*} \rightarrow \mathbb{R}_{+}$is defined by

$$
M\left(t_{0}, \delta\right)=4 n \delta p(n \delta) \text {. }
$$

Thus the inequality $(i)$ is proved.

For the proof of $(i i)$ we consider the function

$$
g: \Delta \rightarrow \mathbb{R}_{+}, g\left(t, t_{0}\right)=\int_{t}^{t+n \delta}\left\|P_{2}\left(u, t_{0}\right) x\right\| d u
$$

where $n$ is a natural number such that $n \delta>4 n\left(t_{0}\right)$. It is easy to see that

$$
g\left(t+2 n\left(t_{0}\right), t_{0}\right) \geq 2 g\left(t, t_{0}\right) \geq g\left(s, t_{0}\right)
$$

for all $s+n\left(t_{0}\right) \geq t \geq s \geq t_{0} \geq 0$.

By Lemma 3.2 we obtain

$$
\begin{aligned}
\int_{s}^{s+\delta}\left\|P_{2}\left(u, t_{0}\right) x\right\| d u & \leq \int_{s}^{s+n \delta}\left\|P_{2}\left(u, t_{0}\right) x\right\| d u \\
& =g\left(s, t_{0}\right) \\
& \leq 4 e^{-v\left(t_{0}\right)(t-s)} \cdot g\left(t, t_{0}\right) \\
& \leq 4 e^{-v\left(t_{0}\right)(t-s)} \cdot\left\|P_{2}\left(t, t_{0}\right) x\right\| \cdot \int_{t}^{t+n \delta}\|P(u, t)\| d u \\
& \leq M\left(t_{0}, \delta\right) e^{-v\left(t_{0}\right)(t-s)}\left\|P_{2}\left(t, t_{0}\right) x\right\|
\end{aligned}
$$

and $(i i)$ is proved. 


\section{The main resuits}

We prove the following

THEOREM 4.1. If the pair $(B, D)$ is admissible for the system ( $P$ ) then there exists an application $N: \mathbb{R}_{+} \rightarrow \mathbb{R}_{+}^{*}$ such that for all $t_{0} \geq 0$ and $u \in B_{t_{0}}(X)$ there is an unique $x_{2}(u) \in X_{2}^{D}\left(t_{0}\right)$ with the properties

(i) $x\left(\cdot, t_{0}, x_{2}(u), u\right) \in D_{t_{0}}(X)$, and

(ii) $\left\|x\left(\cdot, t_{0}, x_{2}(u), u\right)\right\|_{D} \leq N \cdot\|u\|_{B}$.

Proof. By admissiblity of $(B, D)$ for the system $(P)$ it follows that for all $t_{0} \geq 0$ and $u \in B_{t_{0}}(X)$ there is $x_{0} \in X$ such that $x\left(\cdot, t_{0}, x_{0}, u\right) \in D_{t_{0}}(X)$

If we let $x_{k}=P_{k}\left(t_{0}\right) x_{0} \quad(k=1,2)$ then from

$$
x\left(\cdot, t_{0}, x_{0}, u\right)=P\left(\cdot, t_{0}\right) x_{1}+x\left(\cdot, t_{0}, x_{2}, u\right) \in D_{t_{0}}(X)
$$

and $P\left(\cdot, t_{0}\right) x_{1} \in D_{t_{0}}(X)$ it follows that $x\left(\cdot, t_{0}, x_{2}, u\right) \in D_{t_{0}}(X)$ and hence for all $t_{0} \geq 0$ and $u \in B_{t_{0}}(X)$ there exists $x_{2} \in X_{2}^{D}\left(t_{0}\right)$ such that $x\left(\cdot, t_{0}, x_{2}, u\right) \in D_{t_{0}}(X)$.

If we suppose that for $u \in B_{t_{0}}(X)$ there exist $x_{2}^{\prime}, x_{2}^{\prime \prime} \in X_{2}^{D}\left(t_{0}\right)$ such that

$$
x\left(\cdot, t_{0}, x_{2}^{\prime}, u\right) \in D_{t_{0}}(X) \text { and } x\left(\cdot, t_{0}, x_{2}^{\prime \prime}, u\right) \in D_{t_{0}}(X)
$$

then

$$
\begin{aligned}
x\left(\cdot, t_{0}, x_{2}^{\prime}, u\right)-x\left(\cdot, t_{0}, x_{2}^{\prime \prime}, u\right) & =x\left(\cdot, t_{0}, x_{2}^{\prime}-x_{2}^{\prime \prime}, 0\right) \\
& =P\left(t, t_{0}\right)\left(x_{2}^{\prime}-x_{2}^{\prime \prime}\right) \in D_{t_{0}}(X)
\end{aligned}
$$

which implies 


$$
x_{2}^{\prime}-x_{2}^{\prime \prime} \in x_{1}^{D}\left(t_{0}\right) \cap x_{2}^{D}\left(t_{0}\right)=\{0\},
$$

and hence $x_{2}^{\prime}=x_{2}^{\prime \prime}$.

Consider the Banach space $Y_{t_{0}}(X)=X_{2}^{D}\left(t_{0}\right) \times D_{t_{0}}(X)$ with the norm

$$
\left\|\left(x_{2}, d\right)\right\|_{1}=\left\|x_{2}\right\|+\|d\|_{D}
$$

Let $A: B_{t_{0}}(X) \rightarrow Y_{t_{0}}(X)$ be the linear operator defined by

$$
A u=\left(x_{2}(u), x\left(\cdot, t_{0}, x_{2}(u), u\right)\right) .
$$

To establish property ( $i i)$ it is enough by the closed graph theorem to show that $A$ is a closed operator.

Let $u_{n} \rightarrow u$ in $B_{t_{0}}(X)$ and $A u_{n} \rightarrow\left(x_{2}, y\right)$ in $Y_{t_{0}}(X)$. Then $x_{2}\left(u_{n}\right) \rightarrow x_{2}$ in $X_{2}^{D}\left(t_{0}\right)$ and $x\left(\cdot, t_{0}, x_{2}\left(u_{n}\right), u_{n}\right) \rightarrow y$ in $D_{t_{0}}(X)$. From $D(X) \rightarrow L_{\text {loc }}^{1}(X)$ it follows that there exists a subsequence $\left(u_{n_{k}}\right)$ of $\left(u_{n}\right)$ such that

$$
y(t)=\lim _{k \rightarrow \infty} x\left(t, t_{0}, x_{2}\left(u_{n_{k}}\right), u_{n_{k}}\right)
$$

almost everywhere.

From

$\left\|y(t)-x\left(t, t_{0}, x_{2}, u\right)\right\|$

$\leq\left\|y(t)-x\left(t, t_{0}, x_{2}\left(u_{n_{k}}\right), u_{n_{k}}\right)\right\|+\left\|x\left(t, t_{0}, x_{2}\left(u_{n_{k}}\right), u_{n_{k}}\right)-x\left(t, t_{0}, x_{2}, u\right)\right\|$

$\leq\left\|y(t)-x\left(t, t_{0}, x_{2}\left(u_{n_{k}}\right), u_{n_{k}}\right)\right\|+\left\|P\left(t, t_{0}\right)\right\| \cdot\left\|x_{2}\left(u_{n_{k}}\right)-x_{2}\right\|$ $+\int_{t_{0}}^{t}\|P(t, s)\|\left\|u_{n_{k}}(s)-u(s)\right\| d s$

$\leq\left\|y(t)-x\left(t, t_{0}, x_{2}\left(u_{n_{k}}\right), u_{n_{k}}\right)\right\|+p\left(t-t_{0}\right)\left(\left\|x_{2}\left(u_{n_{k}}\right)-x_{2}\right\|+\alpha_{B}(t) \cdot\left\|u_{n_{k}}-u\right\|_{B}\right)$,

for $k \rightarrow \infty$ we obtain that 


$$
y(t)=x\left(t, t_{0}, x_{2}, u\right)
$$

almost everywhere. Hence $x\left(\cdot, t_{0}, x_{2}, u\right) \in D_{t_{0}}(X)$, which implies that $x_{2}=x_{2}(u)$ and

$$
A u=\left(x_{2}(u), x\left(t, t_{0}, x_{2}(u), u\right)\right)=\left(x_{2}, y\right) .
$$

COROLLARY 4.2. If the pair $\left(B, L^{\infty}\right)$ is admissible for the system (P) and

$$
\lim _{t \rightarrow \infty} \alpha_{B}(t)=\infty
$$

then there is $M: \mathbb{R}_{+} \rightarrow \mathbb{R}_{+}^{*}$ such that

$$
\left\|P_{2}\left(t, t_{0}\right) x\right\| \leq M\left(t_{0}\right)\left\|P\left(t+1, t_{0}\right) x\right\|
$$

for azz $\left(t, t_{0}\right) \in \Delta$ and $x \in X$.

Proof. Let $\left(t, t_{0}\right) \in \Delta, x \in X$ and the input function

$$
u: \mathbf{R}_{+} \rightarrow X, u(s)=\varphi_{t+1}^{t+2}(x) P\left(s, t_{0}\right) x
$$

From

$$
\|u(s)\| \leq p(I) \varphi_{t+1}^{t+2}(s) \cdot\left\|P\left(t+1, t_{0}\right) x\right\|
$$

it follows that $u \in B_{t_{0}}(X)$ and

$$
\|u\|_{B} \leq p(1) B_{B}(1) \cdot\left\|P\left(t+1, t_{0}\right) x\right\| \text {. }
$$

From

$$
\begin{aligned}
x\left(s, t_{0},-P_{2}\left(t_{0}\right) x, u\right) & =-P_{2}\left(s, t_{0}\right) x+\int_{t_{0}}^{s} \varphi_{t+1}^{t+2}(u) P\left(s, t_{0}\right) x d u \\
& =\left\{\begin{array}{l}
P_{1}\left(s, t_{0}\right) x, \text { if } s \geq t+2, \\
-P_{2}\left(s, t_{0}\right) x, \text { if } s \leq t+1,
\end{array}\right.
\end{aligned}
$$

it follows that $x\left(\cdot, t_{0},-P_{2}\left(t_{0}\right) x, u\right) \in L_{t_{0}}^{\infty}(X)$.

By Theorem 4.1 there is an application $N: \mathbf{R}_{+} \rightarrow \mathbb{R}_{+}^{*}$ such that 


$$
\begin{aligned}
\left\|P_{2}\left(t, t_{0}\right) x\right\| & \leq N\left(t_{0}\right) \cdot\|u\|_{B} \\
& \leq N\left(t_{0}\right) p(1) B_{B}(1)\left\|P\left(t+1, t_{0}\right) x\right\| \\
& =M\left(t_{0}\right)\left\|P\left(t+1, t_{0}\right) x\right\|
\end{aligned}
$$

for all $\left(t, t_{0}\right) \in \Delta$ and $x \in X$.

THEOREM 4.3. If the pair $\left(L^{1}, L^{\infty}\right)$ is admissible for the system then $P(\cdot, \cdot)$ is a dichotomic process.

Proof. Let $\left(s, t_{0}\right) \in \Delta, x \in X$ and

$$
u(t)=\varphi_{s}^{s+1}(t) P\left(t, t_{0}\right) x
$$

Clearly $u \in L_{t_{0}}^{l}(X)$ and from

$$
x\left(t, t_{0},-P_{2}\left(t_{0}\right) x, u\right)=\left\{\begin{array}{l}
-P_{2}\left(t, t_{0}\right) x, \text { if } t \leq s, \\
P_{1}\left(t, t_{0}\right) x, \text { if } t \geq s+1,
\end{array}\right.
$$

it follows that $x\left(\cdot, t_{0},-P_{2}\left(t_{0}\right) x, u\right) \in L_{t_{0}}^{\infty}(X)$.

By Theorem 4.1 there is $N: \mathbb{R}_{+} \rightarrow \mathbb{R}_{+}^{*}$ such that

$$
\left\|P_{1}\left(t, t_{0}\right) x\right\| \leq N\left(t_{0}\right) p(1) \cdot\left\|P\left(s, t_{0}\right) x\right\| \text { for all } t \geq s+1
$$

and

$$
\left\|P_{2}\left(t, t_{0}\right) x\right\| \leq N\left(t_{0}\right) p(1) \cdot\left\|P\left(s, t_{0}\right) x\right\| \text { for } t \leq s
$$

If $t \in[s, s+1]$ then

$$
\begin{aligned}
\left\|P_{1}\left(t, t_{0}\right) x\right\| & \leq p(1) \cdot\left\|P_{1}\left(s, t_{0}\right) x\right\| \\
& \leq p(1)\left(\left\|P\left(s, t_{0}\right) x\right\|+\left\|P_{2}\left(s, t_{0}\right) x\right\|\right) \\
& \leq M\left(t_{0}\right)\left\|P\left(s, t_{0}\right) x\right\|,
\end{aligned}
$$

where

$$
M\left(t_{0}\right)=\max \left\{N\left(t_{0}\right) p(1), p(1)+N\left(t_{0}\right) p(1)^{2}\right\}
$$

Finally we obtain 


$$
\left\|P_{1}\left(t, t_{0}\right) x\right\| \leq M\left(t_{0}\right)\left\|P\left(s, t_{0}\right) x\right\| \text { for all } t \geq s \geq t_{0} \geq 0
$$

and

$$
\left\|P_{2}\left(t, t_{0}\right) x\right\| \leq M\left(t_{0}\right)\left\|P\left(s, t_{0}\right) x\right\| \text { for } 0 \leq t_{0} \leq t \leq s
$$

These inequalities show that $P(\cdot, \cdot)$ is a dichotomic process.

THEOREM 4.4. Let $(B, D)$ be a pair of Schäffer spaces satisfying Assumption 1 and suppose that Assumption 2 holds. If $P(\cdot, \cdot)$ is $(B, D)$ dichotomic then it is exponentially dichotomic.

Proof. Let $t \geq s \geq t_{0} \geq 0$ and $x \in X$. If $t \geq s+1$ and $u \in[t-1, t]$ then

$$
\left\|P_{1}\left(t, t_{0}\right) x\right\| \leq\|P(t, u)\| \cdot\left\|P_{1}\left(u, t_{0}\right) x\right\| \leq p(t-u)\left\|P_{1}\left(u, t_{0}\right) x\right\| .
$$

By Lemma 3.4 there are $\nu: \mathbb{R}_{+} \rightarrow \mathbb{R}_{+}^{*}, M: \mathbb{R}_{+} \times \mathbb{R}_{+}^{*} \rightarrow \mathbb{R}_{+}$such that

$$
\begin{aligned}
\left\|P_{1}\left(t, t_{0}\right) x\right\| & \leq \int_{t-1}^{t} p(t-u)\left\|P_{1}\left(u, t_{0}\right) x\right\| d u \\
& \leq p(1) \int_{t-1}^{t}\left\|P_{1}\left(u, t_{0}\right) x\right\| \cdot d u \\
& \leq p(1) M\left(t_{0}, 1\right) e^{-v\left(t_{0}\right)(t-s)} \cdot\left\|P_{1}\left(s, t_{0}\right) x\right\|,
\end{aligned}
$$

for $t \geq s+1$ and $s \geq t_{0} \geq 0$.

If $t \in[s, s+1]$ then

$\left\|P_{1}\left(t, t_{0}\right) x\right\| \leq p(t-s)\left\|P_{1}\left(s, t_{0}\right) x\right\| \leq p(1) e^{\nu\left(t_{0}\right)} e^{-\nu\left(t_{0}\right)(t-s)} \cdot\left\|P_{1}\left(s, t_{0}\right) x\right\|$.

Finally we obtain

$$
\left\|P_{1}\left(t, t_{0}\right) x\right\| \leq N_{1}\left(t_{0}\right) e^{-v\left(t_{0}\right)(t-s)}\left\|P_{1}\left(s, t_{0}\right) x\right\|
$$

for all $t \geq s \geq t_{0} \geq 0$ and $x \in X$, where

$$
N_{1}\left(t_{0}\right)=\max \left\{p(1) M t_{0}, 1, p(1) e^{v\left(t_{0}\right)}\right\} \text {. }
$$

Let $m, \delta: \mathbb{R}_{+} \rightarrow \mathbb{R}_{+}^{*}$ given by Assumption 2 and let $\delta_{0}=\delta\left(t_{0}\right)$ and 
$m_{0}=m\left(t_{0}\right)$

Then

$$
m_{0} \cdot\left\|P_{2}\left(s, t_{0}\right) x\right\| \leq\left\|P_{2}\left(s+\delta_{0}, t_{0}\right) x\right\| \leq p\left(s+\delta_{0}-u\right) \cdot\left\|P_{2}\left(u, t_{0}\right) x\right\|
$$

for all $\left(s, t_{0}\right) \in \Delta, u \in\left[s, s+\delta_{0}\right]$ and $x \in X$.

By integration in raport with $u$ on $\left[s, s+\delta_{0}\right]$ from Lemma 3.4 we have

$$
\begin{aligned}
m_{0} \delta_{0}\left\|P_{2}\left(s, t_{0}\right) x\right\| & \leq p\left(\delta_{0}\right) \cdot \int_{s}^{s+\delta_{0}}\left\|P_{2}\left(u, t_{0}\right) x\right\| d u \\
& \leq p\left(\delta_{0}\right) M\left(t_{0}, \delta_{0}\right) \cdot e^{-v\left(t_{0}\right)(t-s)} \cdot\left\|P_{2}\left(t, t_{0}\right) x\right\|,
\end{aligned}
$$

for all $t \geq s \geq t_{0} \geq 0$ and $x \in X$.

THEOREM 4.5. If the pair $(B, D)$ is admissible for the system then the evolutionary process $P(\cdot, \cdot)$ is $(B, D)$ dichotomic.

Proof. Let $t_{0} \geq 0, \delta>0$ and $x \in X$ such that

$$
P_{1}\left(t, t_{0}\right) x \neq 0 \text { for all } t \geq t_{0} \text {. }
$$

Then $P\left(t, t_{0}\right) \neq 0$ for every $t \geq t_{0}$.

For each $\left(t, t_{0}\right) \in \Delta$ we consider the function

$$
\begin{aligned}
& u_{0}(s)= \begin{cases}\frac{P\left(s, t_{0}\right) x}{\left\|P\left(s, t_{0}\right) x\right\|}, & \text { if } s \in[t, t+\delta], \\
0 & , \text { if } s \notin[t, t+\delta],\end{cases} \\
& =\frac{\varphi_{t}^{t+\delta}(s) P\left(s, t_{0}\right) x}{\left\|P\left(s, t_{0}\right] x\right\|} \text {. }
\end{aligned}
$$

From

$$
\left\|u_{0}(x)\right\| \leq \beta_{B}(\delta)
$$

it results that $u_{0} \in B_{t_{0}}(X)$ and $\left\|u_{0}\right\|_{B} \leq B_{B}(\delta)$. If 


$$
f\left(t, t_{0}\right)=\int_{t}^{t+\delta} \frac{d s}{\left\|P\left(s, t_{0}\right) x\right\|},
$$

and $x_{0}=-f\left(t, t_{0}\right) P_{2}\left(t_{0}\right) x$ then

$$
x\left(s, t_{0}, x_{0}, u_{0}\right)=\left\{\begin{array}{l}
f\left(t, t_{0}\right) P_{1}\left(s, t_{0}\right) x, \text { if } s \geq t+\delta, \\
-f\left(t, t_{0}\right) P_{2}\left(s, t_{0}\right) x, \text { if } t_{0} \leq s \leq t,
\end{array}\right.
$$

and hence $x\left(\cdot, t_{0}, x_{0}, u_{0}\right) \in D_{t_{0}}(X)$.

Since $x_{0} \in x_{2}^{D}\left(t_{0}\right)$, by Theorem 4.1 we have that there is $\boldsymbol{N}: \mathbf{R}_{+} \rightarrow \mathbf{R}_{+}^{*}$ such that

$$
\left\|x\left(\cdot, t_{0}, x_{0}, u_{0}\right)\right\|_{D} \leq N\left(t_{0}\right) \cdot\left\|u_{0}\right\|_{B} \leq N\left(t_{0}\right) B_{B}(\delta)
$$

From here, Remark 2.4 and by Schwartz's inequality we obtain $\left\|\varphi_{t+\delta}(\cdot) P_{1}\left(\cdot, t_{0}\right) x\right\|_{D}+\left\|\varphi_{t_{0}}^{t}(\cdot) P_{2}\left(\cdot, t_{0}\right) x\right\|_{D}$

$$
\begin{aligned}
& \leq \frac{2 N\left(t_{0}\right) B_{B}(\delta)}{f\left(t, t_{0}\right)} \\
& \leq \frac{2 N\left(t_{0}\right) B_{B}(\delta)}{\delta^{2}} \cdot \int_{t}^{t+\delta}\left\|P\left(s, t_{0}\right) x\right\| d s \\
& \leq \frac{4 N\left(t_{0}\right)}{\delta \alpha_{B}(\delta)} \cdot \int_{t}^{t+\delta}\left\|P\left(\tau, t_{0}\right) x\right\| d \tau .
\end{aligned}
$$

If for $x \in X$ there is $t_{1} \geq t_{0}$ such that $P\left(t_{1}, t_{0}\right) x=0$ then $P\left(t, t_{0}\right) x=P\left(t, t_{1}\right) P\left(t_{1}, t_{0}\right) x=0$ for all $t \geq t_{1}$ and thus $x \in x_{1}^{D}\left(t_{0}\right)$, which implies $x=P_{1}\left(t_{0}\right) x$.

Let $\tau$ be a positive number such that $P\left(\tau, t_{0}\right) x=0$ and $P\left(t, t_{0}\right) x \neq 0$ for every $t \in\left[t_{0}, \tau\right]$.

Let $t \geq t_{0}, \delta>0, t_{2} \in(t, t+\delta]$ and $\delta_{2}>0$ such that $t+\delta_{2}<t_{2}$. If $t+\delta<\tau$ then we consider the input function defined by 


$$
u(s)= \begin{cases}\frac{P\left(s, t_{0}\right) x}{\left\|P\left(x, t_{0}\right) x\right\|}, \text { if } s \in\left[t, t_{2}-\delta_{2}\right], \\ \frac{P\left(s, t_{0}\right) x}{\left\|P\left(t_{2}-\delta_{2}, t_{0}\right)\right\|}, & \text { if } s \in\left[t_{2}-\delta_{2}, t_{2}\right], \\ 0, & \text { if } s \leqslant\left[t, t_{2}\right] .\end{cases}
$$

From Remarks 2.1 and 2.4 it follows that there exists $\omega>0$ such that

$$
\|u\|_{B} \leq e^{\omega \delta} 2 \cdot \beta_{B}(\delta)
$$

Hence $u \in B_{t_{0}}(X)$ and

$$
x\left(s, t_{0}, 0, u\right)=\left(\int_{t}^{t_{2}-\delta_{2}} \frac{d u}{\left\|P_{1}\left(u, t_{0}\right) x\right\|}+\frac{\delta_{2}}{\left\|P_{1}\left(t_{2}-\delta_{2}, t_{0}\right) x\right\|}\right) \cdot P_{1}\left(s, t_{0}\right) x
$$

for all $s \geq t_{2}$.

Because $0 \in X_{2}^{D}\left(t_{0}\right)$ and $x\left(\cdot, t_{0}, 0, u\right) \in D_{t_{0}}(x)$ from Theorem 4.1 we obtain that there is $N: \mathbb{R}_{+} \rightarrow \mathbb{R}_{+}^{*}$ such that

$$
\left\|x\left(\cdot, t_{0}, 0, u\right)\right\|_{D} \leq N\left(t_{0}\right) \beta_{B}(\delta) e^{\omega \delta_{2}} \text {. }
$$

Hence

$$
\left\|\varphi_{t_{2}}(\cdot) P_{1}\left(\cdot, t_{0}\right) x\right\|_{D} \cdot \int_{t}^{t_{2}-\delta_{2}} \frac{d u}{\left\|P_{1}\left(u, t_{0}\right) x\right\|} \leq N\left(t_{0}\right) \beta_{B}(\delta) e^{\omega \delta_{2}}
$$

for every $\delta_{2}>0$ with $t+\delta_{2}<t_{2}$.

If $t_{2}=t+\delta$ and $\delta_{2} \rightarrow 0$ then

$$
\left\|\varphi_{t+\delta}(\cdot) P_{1}\left(\cdot, t_{0}\right) x\right\|_{D} \cdot \int_{t}^{t+\delta} \frac{d u}{\left\|P_{1}\left(u, t_{0}\right) x\right\|} \leq N\left(t_{0}\right) \beta_{B}(\delta) .
$$

As in the preceding case (using Remark 2.4 and Schwartz's inequality) we obtain 


$$
\left\|\varphi_{t+\delta}(\cdot) P_{1}\left(\cdot, t_{0}\right) x\right\| \leq \frac{2 N\left(t_{0}\right)}{\delta \alpha_{B}(\delta)} \cdot \int_{t}^{t+\delta}\left\|P\left(u, t_{0}\right) x\right\| d u
$$

If $t+\delta \geq \tau$ this inequality remains obviously true. Thus

$$
\begin{aligned}
\left\|\varphi_{t+\delta}(\cdot) P_{1}\left(\cdot, t_{0}\right) x\right\|_{D} & =\left\|\varphi_{t+\delta}(\cdot) P_{1}\left(\cdot, t_{0}\right) x\right\|_{D}+\left\|\varphi \varphi^{t}(\cdot) \cdot P_{2}\left(\cdot, t_{0}\right) x\right\|_{D} \\
& \leq \frac{2 N\left(t_{0}\right)}{\delta \alpha_{B}(\delta)} \cdot \int_{t}^{t+\delta}\left\|P\left(u, t_{0}\right) x\right\| d u,
\end{aligned}
$$

for all $\left(t, t_{0}\right) \in \Delta, \delta>0$ and $x \in X$. This shows that $P(\cdot, \cdot)$ is $(B, D)$ dichotomic.

COROLLARY 4.6. Let $(B, D)$ be a pair of Schäffer spaces satisfying Assumption 1 and suppose that Assumption 2 holds.

If the pair $(B, D)$ is admissible for the system $(P)$ then the process $P(\cdot, \cdot)$ is exponentially dichotomic.

Proof. This is a consequence of Theorems 4.4 and 4.5 .

COROLLARY 4.7. Let $P(\cdot, \cdot)$ be an evolutionary process satisfying Assumption 2. If

(i) $B=L^{p}, p>1$ or $B=M^{p}, p \geq 1$ or $B=C$ and $D \in S$, or

(ii) $B \in S$ and $D=L^{q}, q<\infty$ or $D=C$

and $(B, D)$ is admissible for the system $(P)$ then $P(\cdot, \cdot)$ is an exponential dichotomic process.

Proof. In the above hypotheses we have (see [3], pp. 61-67 that the pair $(B, D)$ verifies Assumption 1 . Then the result is obvious from the preceding corollary.

COROLLARY 4.8. If $\lim _{t \rightarrow \infty} \alpha_{B}(t)=\infty$ and the pair $\left(B, L^{\infty}\right)$ is admissible for the system $(P)$ then the evolutionary process $P(\cdot, \cdot)$ is exponentially dichotomic.

Proof. This results from Remark 2.7 and Corollary 4.6. 


\section{References}

[1] W.A. Coppel, Dichotomies in stability theory (Lecture Notes in Mathematics, 629. Springer-Verlag, Berlin, Heidelberg, New York, 1978).

[2] Ruth Curtain and A.J. Pritchard, "The infinite-dimensional Riccati equation for systems defined by evolution operators", SIAM J. Control Optim. 14 (1976), 951-983.

[3] José Luis Massera, Juan Jorge Schäffer, Linear differential equations and function spaces (Pure and Applied Mathematics, 21. Academic Press, New York and London, 1966).

[4] Mihail Megan, "On the input-output stability of linear controllable systems", Canad. Math. Bull. 21 (1978), 187-195.

[5] Mihail Megan and Petre Preda, "On exponential dichotomy in Banach spaces", Bulz. Austral. Math. Soc. 23 (1981), 293-306.

[6] Kenneth J. Palmer, "Two linear systems criteria for exponential dichotomy", Ann. Mat. Pura Appl. (to appear).

[7] Luciano Pandolfi, "Exponential stability and Liapunov equation for a class of functional differential equations", Boll. Mat. Ital. (5) 168 (1979), 897-909.

[8] M. Региш [M. Regiš], "К неравномерной асимттотической уСТОЙЧИВОстИ" [On nonuniform asymptotic stability], Prikl. Mat. Meh. 27 (1963), 23l-243. English Transl: J. Appl. Math. Mech. 27 (1963), 344-362.

Department of Mathematics, University of Timişoara, 1900 - Timişoara, R.S. România. 